\title{
Synchronization Problems on Small-world Networks with time delay
}

\author{
Lei $\mathrm{GU}^{1,}$, , Jinjin $\mathrm{SHI}^{2, \mathrm{~b}}$
}

${ }^{1}$ Shanghai Research Institute of China Telecom Corporation Limited, Shanghai, 200122,China

${ }^{2}$ Shanghai Hyron Software, Shanghai, 200231,China

aemail: email:gehirn@sina.com, bemail: shijinjincg@163.com

Keywords: Synchronization Problem; Small-world Network; Time Delay;

\begin{abstract}
In this paper, we study the synchronization problem on small-world network with time delay. In particular, we give rigorous mathematical analysis and arrive with sufficient condition of the synchronization problem when the synchronous states are global stable. The general closed-form analytical results build the relationship between network topological characteristic and the network dynamical characteristic of time delay.
\end{abstract}

\section{Introduction}

Synchronization problem for a coupled dynamics system origins from the synchronization phenomenon in physics and biology. The study can be traced back to the Kuramoto system [1], which is now a research area on whether coupled agents with continuous and discrete time chaos, circuit as well as bifurcation systems are stable or not [2] [3] [4] [5] [6] [7] [8] [9] [10] [11]. The stability of coupled system is related to both network topology and the dynamic system of individual agents. In [4] [12] [13] [14] [15], different sufficient conditions are investigated for the synchronization problem.

Synchronization problem in small-world networks has been carefully studied [16] [17] [18] [19] [20]. It has been observed that the ability to archive synchronization is greatly enhanced by making the network become a small-world network. Small-world networks are those network that exhibit two important characterizations: i.e., large clustering coefficient, and the small world phenomenon (small diameter, or short average distance) in the real complex networks. Watts and Strogatz [21] in 1998 proposed a famous small-world network model which possesses both small average distance and large clustering coefficient by simulation. Newman and Watts proposed another model [22] satisfying the two properties which is the union of an E-R random graph[23] and a $2 \mathrm{k}$ regular cycle. $\mathrm{Gu}$, Huang and Zhang further introduced a generalized small-world model [24] to cover both Watts-Strogatz model and Newman-Watts model.

In this paper, we study the synchronization problem on Newman-Watts type small-world network with time delay. In particular, we give the sufficient condition of the synchronization problem based on linear feedback controllers which can also be applied in the situation without feedback as the special case. Our result provides a general closed-form analytical result that builds the relationship between network topological characteristic parameters and the network dynamical characteristic parameter of time delay.

\section{Network Dynamics and Spectral Analysis of Newman-Watts Network}

Suppose there are $\mathrm{N}$ nodes (oscillators) and $\mathbf{x}^{\mathrm{i}}$ be the $\mathrm{m}$-dimensional vector of dynamical variables of the $i_{\text {th }}$ node. The dynamics of the $i_{\text {th }}$ node are

$$
\dot{x}^{i}(t)=F\left[x^{i}(t)\right]+\sigma \sum_{j} L_{i j} H x^{j}(t-\tau)
$$

where $\sigma$ is a coupling strength and $L$ is a Laplacian matrix. Synchronization on such a network means when time is long enough, $\lim _{t \rightarrow \infty} x_{1}(t)=\ldots=\lim _{t \rightarrow \infty} x_{n}(t)$. This defines the synchronization manifold. Pecora and Carroll shows that it is a invariant manifold with some additional assumptions and therefore it can apply linear approximation near synchronization state. And therefore one get 
the corresponding variational equation, here $D F$ and $D H$ are correspondent Jacobian matrix of $F$ and $H$.

$$
\dot{\xi}^{i}(t)=D F\left[\xi^{i}(t)\right]+\sigma \sum_{j} L_{i j} D H \xi^{j}(t-\tau)
$$

Supposing the small-world network model is an undirected graph. With matrix transformation we can diagonalize $L$, the matrix is symmetric and hence the eigenvalues are all real, denoted by $\lambda_{k}, k=1, \ldots n$. Then the stability of Equation (2) is equivalent to the stability of the stability of $n$ collection of following equations.

$$
\dot{\xi}^{i}(t)=D F\left[\xi^{i}(t)\right]+\sigma \lambda_{k} D H \xi^{j}(t-\tau)
$$

Therefore arrive with the following result[13]:

Suppose the $\mathrm{n}-1$ system are all asymptotically stable, that

$$
\dot{\xi}^{i}(t)=D F\left[\xi^{i}(t)\right]+\sigma \lambda_{k} D H \xi^{j}(t-\tau)
$$

for all $\lambda_{k}, k=2, \ldots n$. Where $0=\lambda_{1} \leq \lambda_{2} \leq \ldots \leq \lambda_{n}$ are the eigenvalues of Laplacian matrix, then the coupled system is also asymptotically stable. Here the stability means local stable near the synchronous states.

Moreover, Wu and Jiao [15] proposed a time-delay coupled system with linear feedback controllers

$$
\dot{X}(t)=F[X(t)]+\sigma L \times A(t-\tau)+\kappa_{n} \times A(X(t)-S(t))
$$

where $\mathrm{S}(\mathrm{t})$ is the synchronous state of the system. Moreover it is assuming that there exists $\bar{x}(t)$ so that $f(x(t))-f(y(t))=D f(\bar{x}(t))$, where $D f$ could be the Jacobian $D F$ or other related matrix [15].

Wu and Jiao [15] give the sufficient condition of global stablity, exists $\varepsilon>0$

$$
D f[\bar{x}(t)]^{T}+D f[\bar{x}(t)]-\kappa\left(A^{T}+A\right)+\lambda_{n}^{2} A A^{T} \prec-(\varepsilon+1) / \sqrt{N} I_{n}
$$

Here matrix $A \prec B$ means that matrix $\mathrm{B}-\mathrm{A}$ is nonnegative definite matrix.

Equation (6) implies that the sufficient condition is determined by the largest eigenvalue of the Laplacian, especially for Newman-Watts small-world network we have the following result.

Lemma 1: Let $\mathrm{NW}(\mathrm{n}, \mathrm{c}, \mathrm{k})$ be the Newman-Watts network of n nodes which is the union of

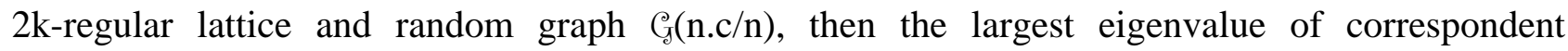
Laplacian is $(2+o(1)) \log n / \log \log n$.

Proof:

In [18] Equation (8) shows that the maximum degree of $N W(n, c, k)$ is $(1+o(1)) \log n / \log \log n$. Moreover Lemma 2.4 in [18] shows that the largest eigenvalue is smaller than two times maximum degree, and the result follows directly.

Now we are able to arrive with our main result as follows

Theorem 1: Consider the time-delayed coupled system $\dot{X}(t)=F[X(t)]+\sigma L \times A(t-\tau)+\kappa_{n} \times A(X(t)-S(t))$, where $\mathrm{L}$ is the Laplacian matrix of Newman-Watts network NW(n,c,k). Letting $B(\varepsilon)=\left[\kappa\left(A^{T}+A\right)-(\varepsilon+1) / \sqrt{N} I_{n}-D f[\bar{x}(t)]^{T}-D f[\bar{x}(t)]\right]\left(A^{T}\right)^{-1} A^{-1}$, then the synchronous state of dynamic system is global stable if $\lambda_{\min }(B(\varepsilon)) \geq(4+o(1)) \log ^{2} n / \log ^{2} \log ^{2} n$.

Proof:

Rewrite equation (6) becomes $\lambda_{n}^{2} A A^{T} \prec \kappa\left(A^{T}+A\right)-(\varepsilon+1) / \sqrt{N} I_{n}-D f[\bar{x}(t)]^{T}-D f[\bar{x}(t)]$. Multiply both side by $\left(A^{T}\right)^{-1} A^{-1}$, the equation becomes $\lambda_{n}^{2} I_{N} \prec B(\varepsilon)$. Remember that by Lemma 1, $\lambda_{n} \leq(2+o(1)) \log n / \log \log n$ and B is symmetric matrix, the result follows directly.

Theorem 1 provides a general closed-form analytical result that builds the relationship between network topological characteristic parameters and the network dynamical characteristic parameter of time delay. Moreover, setting $\kappa=0$, equation (5) degenerates into equation (1) so that the 
situation without feedback play as a special case.

\section{Conclusion}

In this paper, we study the synchronization problem on small-world network with time delay. In particular, we give rigorous mathematical analysis and arrive with sufficient condition of the synchronization problem when the synchronous states are global stable. The general closed-form analytical results build the relationship between network topological characteristic and the network dynamical characteristic of time delay.

\section{Acknowledgement}

In this paper, the research was supported by the Ministry of Science and Technology under the grant MOST 103-2221-E-002-022-MY3, the Major Research Plan of the National Natural Science Foundation of China under Grant 91118008, and by Key Projects in the National Science \& Technology Pillar Program during the Twelfth Five-year Plan Period under Grant 2014BAC16B01.

\section{References}

[1] Y. Kuramoto, Cooperative dynamics of oscillator community - a study based on lattice of rings, in International Symposium on Mathematical Problems in Theoretical Physics, edited by H. Araki, Lecture Notes in Physics, 39,1975, Springer, Berlin,

[2] F. M. Atay, J. Jost and A. Wende, Delays, Connection Topology, and Synchronization of Coupled Chaotic Maps, Phys. Rev. Lett., 92,2004, 144101.

[3] F. M. Atay, T. Biyikoglu and J. Jost, Network synchronization: Spectral versus statistical properties, Physica D, 224,2006, 35-41.

[4] M. Barahona and L. M. Pecora, Synchronization in small-world systems, Phys. Rev. Lett.,89,2002, 054101.

[5] J. Fan and X. F. Wang, On synchronous preference of complex dynamical networks, Phys. A, 355,2005, 657-666.

[6] D. J. Gauthier and J. C. Bienfang, Intermittent Loss of Synchronization in Coupled Chaotic Oscillators: Toward a New Criterion for High-Quality Synchronization, Phys. Rev. Lett., 77,1996, 1751-1754.

[7] J. F. Heagy, T. L. Carroll, and L. M. Pecora, Short wavelength bifurcations and size instabilities incoupled oscillators systems, Phys. Rev. Let, 73,1995, 4185-4188.

[8] J. F. Heagy, T. L. Carroll, and L. M. Pecora, Synchronous chaos in coupled oscillator systems, Phys. Rev. E, 50,1994, 1874-1885.

[9] J. M. Kowalski, G. L. Albert, and G. W. Gross, Asympotically synchronous orbits in systems of excitable elements, Phys. Rev. A, 42,1990, 6260-6263.

[10] L. M. Pecora, T. L. Carroll, and J. F. Heagy, Synchronous chaos in coupled oscillator systems, Phys. Rev. E, 52,1995, 3420-3439.

[11] L. M. Pecora and T. L. Carroll, Master Stability Functions for Synchronized Coupled Systems, Phys. Rev. Lett, 80,1998, 2109-2113.

[12] K. S. Fink, G. Johnson, T. Carroll, D. Mar, and L. M. Pecora, Three coupled oscillators as a universal probe of synchronization stability in coupled oscillator arrays, Phys. Rev. E, 61,2000, 5080-5090 
[13] C. G. Li, G. Chen, Synchronization in general complex dynamical networks with coupling delays, Phys. A, 343, 2004, 236-278.

[14] W. L. Lu, T. P. Chen, G. Chen, Synchronization analysis of linearly coupled systems described by differential equations with a coupling delay, Phys. D, 221,2006, 118-134.

[15] J. Wu and L. Jiao, Synchronization in dynamic networks with nonsymmetrical time-delay coupling based on linear feedback controllers, Phys. A, 387,2008, 2111-2119.

[16] L. F. Lago-Fernandez, R. Huerta, F. Corbacho and J. A. Siguenza, Fast response and temporal coherent oscillations in small-world networks, Phys. Rev. Lett., 84,2000, 2758-2761.

[17] H. Hong, M. Y. Choi and B. J. Kim, Synchronization on small-world networks, Phys. Rev. E, 65,2002, 026139.

[18] L. Gu, X. D. Zhang and Q. Zhou, Consensus and synchronization problems on small-world networks, J. Math. Phys., 51(8),2010, 082701.

[19] C. G. Li, G. Chen, Phase synchronization in small-world networks of chaotic oscillators, Phys. A, 341,2004, 73-79.

[20] X. F. Wang and G. Chen, Synchronization in small-world dynamical networks,Int. J. Bifurcation and Chaos, 12,2002, 187-192.

[21] D. J. Watts and S. H. Strogatz, Collective dynamics of "small world networks", Nature, 393,1998,440-442.

[22] M. E. J. Newman and D. J. Watts, Renormalization group analysis of the small-world network model, Phys. Lett. A, 263,1999, 341-346.

[23] P. Erdös and A. Rényi, On the evolution of random graphs, Pub. Math. Inst. Hungarian Academy of Science, 5,1960, 17-61..

[24] L. Gu, H. L. Huang. and X. D. Zhang, The Clustering Coefficient and the Diameter of Small-world Networks, Acta Mathematica Sinca, English Series,2013,29(1):199-208. 